\title{
Retos y perspectivas en la comunicación organizacional
}

\section{Challenges and perspectives in organizational communication}

\author{
Leticia Rodríguez-Fernández; Pablo Vázquez-Sande
}

Cómo citar este artículo:

Rodríguez-Fernández, Leticia; Vázquez-Sande, Pablo (2019). "Retos y perspectivas en la comunicación organizacional". El profesional de la información, v. 28, n. 5, e280501.

https://doi.org/10.3145/epi.2019.sep.01

Artículo recibido el 02-09-2018

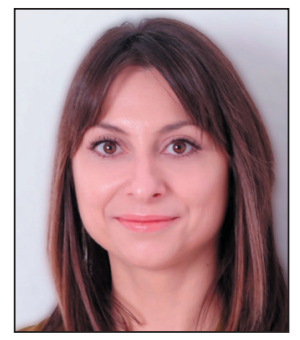

Leticia Rodríguez-Fernández $\triangle$ https://orcid.org/0000-0002-7472-5472

Universidad Nebrija, Facultad de Comunicación y Artes, Departamento de Publicidad

Campus Madrid-Princesa

Sta. Cruz de Marcenado, 27.

28015 Madrid, España

Irodrigu@nebrija.es

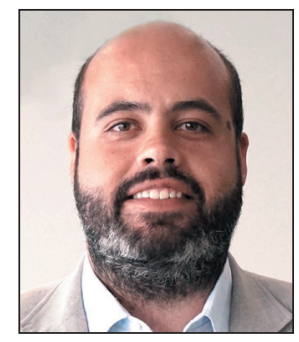

Pablo Vázquez-Sande

http://orcid.org/0000-0001-5081-385X

Universidade de Santiago de Compostela Departamento Ciencias da Comunicación Campus Norte, Avda. de Castelao, $\mathrm{s} / \mathrm{n}$. 15782 Santiago de Compostela (A Coruña), España

pablo.vazquez.sande@usc.es

\section{Resumen}

La comunicación organizacional atraviesa un momento de cambio en el que la tecnología ha asumido un papel protagonista. Su inclusión simplifica y mejora algunos procesos de trabajo pero motiva reflexiones en torno al uso de los datos, la honestidad de las informaciones, la transparencia de las nuevas influencias o la medición de resultados. Se añade la capacidad y necesidad de los profesionales de integrar nuevas competencias en su desempeño; la cooperación de perfiles interdisciplinares que aporten valor; y la inclusión de estos escenarios en la formación universitaria. Se propone una reflexión sobre 1) el impacto de la tecnología: retos derivados y su impacto disruptivo en el sector; 2 ) nuevas influencias en las que la confianza, la transparencia y los resultados están siendo cuestionados; 3) la adaptación de las competencias profesionales a través del engranaje de los profesionales y la Academia; y 4) los desafíos presentes en la investigación en comunicación organizacional.

\section{Palabras clave}

Comunicación organizacional; Comunicación corporativa; Relaciones públicas; Tecnología; Tendencias; Investigación; Estudios; Profesión.

\begin{abstract}
Organisational communication is going through a time of change in which technology has taken on a leading role. Its inclusion simplifies and improves some work processes but motivates reflections on the use of data, the accuracy of the information, the transparency of new influences or the measurement of results. It adds the capacity and need of professionals to integrate new skills in their performance; the cooperation of interdisciplinary profiles that provide value; and the inclusion of these scenarios in university training. A reflection is proposed on 1) the impact of technology: derived challenges and their disruptive impact on the sector; 2) new influences: in which trust, transparency and outcome are being questioned; 3 ) the adaptation of professional competencies through the network of professionals and the Academy; and 4) the challenges present in organizational communication research.
\end{abstract}

\section{Keywords}

Organizational communication; Corporate communication; Public relations; Technology; Trends; Research; University studies; Profession. 


\section{Introducción}

La comunicación organizacional es un área fundamental que ha ido ganando peso en los últimos años, posicionándose como una de las actividades con mayor crecimiento en empleabilidad. El debate sectorial ha dejado de estar tan centrado en el papel de sus responsables pasando a poner el foco en el contexto económico, tecnológico y social, y las perspectivas para seguir fortaleciendo la profesión. Ciertamente, en los últimos años los profesionales del área han asumido mayor número de responsabilidades y competencias al enfrentarse a una realidad voluble cuyos cambios han sido aún más vertiginosos con el rápido desarrollo de la tecnología y su influencia transversal. La promesa de una comunicación personalizada es ya una realidad que ofrece oportunidades para áreas complejas como la comunicación de crisis pero que genera debates sobre la privacidad de los datos generados por los usuarios.

\section{El impacto de la tecnología: retos y disrupción}

La tecnología ha sido un elemento disruptivo en el ámbito de la comunicación, de modo que la mayoría de profesionales y organizaciones sectoriales la identifican como protagonista de las tendencias en los próximos años. El $61 \%$ de los profesionales que trabajan en agencias de comunicación y el 53\% de responsables del área señalan que en los próximos 5 años el cambio en la industria de las relaciones públicas será "considerable". Igualmente, el 59\% considera que la tecnología conducirá este cambio (Global communication report, 2019)

Estas tendencias también se recogen en las perspectivas ofrecidas por las asociaciones españolas. El Anuario de la comunicación 2018 identifica entre los futuros retos profesionales el papel de la inteligencia artificial, la posverdad, la comunicación visual, el management, y la integración del marketing y la comunicación. En la misma línea, la quinta edición del informe El Estado de la comunicación en España (2018) destaca que en los próximos tres años se tendrá que abordar el uso de los datos masivos y los algoritmos para la comunicación $(50,4 \%)$, la conexión de las estrategias de la organización y la comunicación (46,8\%), la creación y gestión de contenidos de calidad que resulten atractivos para los públicos de la organización $(40,1 \%)$ o el impulso de la comunicación interna $(36,2 \%)$, entre otras cuestiones.

La inteligencia artificial, fundamentalmente a través del uso de bots, software que se utiliza para resolver tareas repetitivas, prácticamente automáticas en internet, se ha incorporado a actividades desarrolladas hasta el momento de manera manual. Su sofisticación dependerá de su evolución: algunos sólo entienden órdenes y conceptos simples mientras que otros son capaces de imitar el lenguaje humano incluso en conversaciones. Así, se han agilizado servicios como los chatboxs o el envío de emails comerciales, pero también han motivado un debate público sobre su uso para incrementar el tráfico o ampliar la conver-

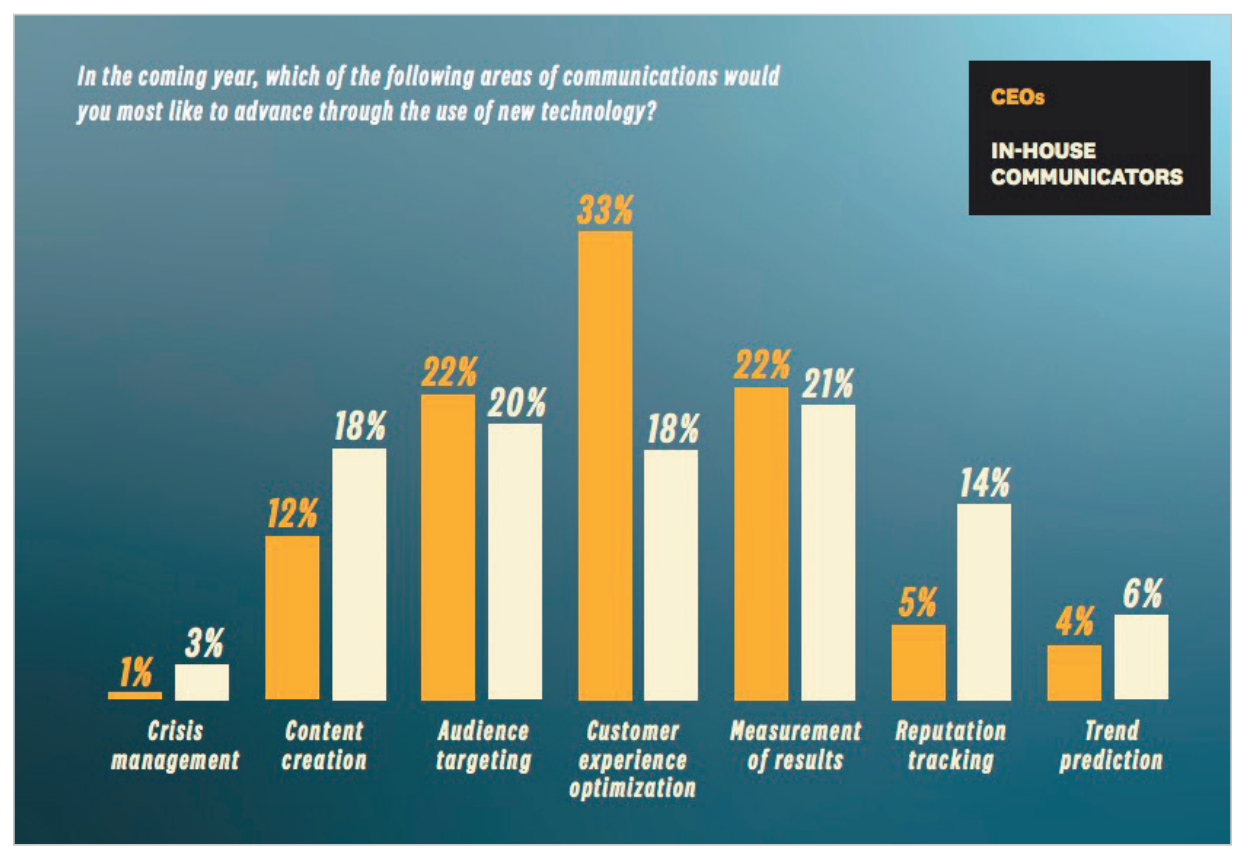

Figura 1. Áreas de la Comunicación que experimentarán más cambio gracias al uso de nuevas tecnologías según las respuestas de CEOs

Fuente: Global communication report 2019 sación social.

Los bots suponen una oportunidad para agilizar algunos procesos comunicativos como la atención al cliente, la conversación y la interacción social o la creación y difusión de contenidos. En España su uso es aún muy reducido en comparación con otros países. Se ha observado su aplicación en la creación de contenidos periodísticos (Túñez-López; Toural-Bran; Cacheiro-Requeijo; 2018) pero aún no se conocen investigaciones o estudios relacionados con la creación de contenidos corporativos. Los profesionales entrevistados en Global communication report (2019) identificaban los informes financieros o las coberturas deportivas como contenidos potenciales que podrían ser redactados por inteligencia artificial.

Estas herramientas, combinadas con el uso de datos, personalizan la comunicación a través del comportamiento de la audiencia, promesa que en los últimos años había sido abordada fundamentalmente desde la microsegmentación publicitaria. Su aplicación en la comunicación organizacional resulta especialmente útil y valiosa en la comunicación 
de crisis y emergencias, al posibilitar, entre otras cuestiones, la respuesta automática y personalizada en función de la geolocalización (Hofeditz et al., 2019).

Otro de los campos en los que la comunicación algorítmica ha tenido gran aceptación y rápida acogida es en la comunicación política, al incorporarse de manera paulatina como un instrumento más de campaña que facilita un alto impacto en la audiencia (Campos-Domínguez; García-Orosa, 2018). La comunicación digital ha dado paso a estrategias más complejas y refinadas que en ocasiones viran hacia la propaganda computacional, entendiendo ésta como

"el conjunto de prácticas ejecutadas por programas informáticos para persuadir de las bondades de ideas, personas o iniciativas" (Redondo-Escudero, 2016).

En este caso los bots se incorporan como herramienta fundamental en la difusión de contenidos, reavivando el debate sobre la necesidad de que la comunicación se asiente en la ética y la honestidad. Del mismo modo, el uso de los datos para fines políticos y/o comerciales precisa aún de una profunda reflexión en torno a la privacidad de los usuarios y los límites derivados de ella (Wiencierz; Röttger, 2019). Se añade la imitación del comportamiento social de alto impacto e influencia en las percepciones de los consumidores, en el discurso público de las organizaciones y en la configuración de la opinión pública.

\section{Nuevas influencias: confianza, transparencia y resultados}

El desarrollo y universalización de los smartphones incentiva un mayor consumo de contenido audiovisual. Según datos de Cisco (2019), el tráfico de vídeo por internet se cuadriplicará de 2017 a 2022 y las emisiones en directo representarán el $17 \%$ de todo el tráfico audiovisual en 2022. Las nuevas estrategias en comunicación compiten por atraer la atención de una audiencia cada vez más saturada de impactos informativos.

El dominio de la cultura audiovisual se refleja en las audiencias en redes sociales. Instagram y YouTube son plataformas hegemónicas de influencia, escaparates de marketing que han cambiado los principios básicos de la prescripción clásica en la sociedad de la transparencia (Han, 2013). Los antiguos líderes de opinión (periodistas, referentes culturales, profesores, políticos...) conviven con nuevos influencers que vehiculan sus ideas por sí mismos, saltando con ello los filtros editoriales y otras limitaciones de los medios de comunicación, quebrando así los roles hegemónicos clásicos de formación de opinión.

Esta influencia se asienta sobre unos cimientos opacos: resulta complicado identificar cuándo una opinión es plenamente sincera o responde a determinados intereses. Por un lado, los influencers reciben gratificaciones más allá de las económicas como el posicionamiento social o el acceso a determinado espacios, circunstancias y experiencias que pueden ser vistas como una oportunidad a la que se accede a través de la opinión. Las "ventanas de poder" se extienden a los prosumidores que, como creadores de contenido, entienden que también pueden utilizar éste para sacar beneficio o perjudicar a la competencia. Por otro lado, la democratización de la opinión ha contribuido a que las organizaciones se acerquen a sus públicos a través de intereses reales, creando comunidad desde sus propias percepciones.

Las comunidades aumentan su valor cuando la microinfluencia comienza en el interior de la organización, convirtiendo al talento en embajador y portavoz más creíble, en algunos contextos, que las fuentes oficiales. A ella se añade la necesidad de que las organizaciones se erijan en líderes de influencia tanto en el sector en el que operan como en lo social. Estos compromisos imprimen identidad a las organizaciones y contribuyen notablemente a que la reputación tenga la fundamental carga de responsabilidad social inherente. Se generan por tanto "comunidades de influencia", en las que los públicos se agrupan por sus intereses y preocupaciones sociales y se retroalimentan positivamente con las conductas responsables y positivas de las organizaciones. Se busca generar confianza a través del diálogo y la transparencia, valor en alza en un contexto marcado por la posverdad y las fake news, ambos erigidos como herramientas para desviar la atención por parte de las élites (Gracia, 2017).

El informe European communication monitor (Zerfass et al., 2019) revela que las cuestiones más estratégicas en la gestión de la comunicación a abordar antes de 2022 serán la creación y mantenimiento de confianza, lidiar con la velocidad y volumen del flujo informativo y explorar nuevas vías para crear y distribuir el contenido. Todos estos retos convergen en internet, que, como espacio informativo, anticipa nuevos debates y desafíos en torno al control del medio, su posible manipulación y sus efectos en la democracia. Malinterpretada, la falsa transparencia se convierte en populismo y contribuye en la creación de un nuevo liderazgo hiperpersonalista, despreocupado de su influencia e impacto social y centrado en el efectivisimo cortoplacista.
Se busca generar confianza, a través del diálogo y la transparencia, valor en alza en un contexto marcado por la posverdad y las fake news 
Los indicadores son, por tanto, las únicas herramientas para demostrar que la influencia, la confianza y la transparencia se traducen en datos reales. Se evidencia la necesidad de ofrecer, desde la práctica de la comunicación, resultados útiles para las organizaciones. Mediciones y técnicas de investigación que ofrezcan valor al área, dado el contexto actual en el que la interdisciplinariedad desdibuja las diferencias entre la publicidad, el marketing y la comunicación corporativa. La medición de resultados es una oportunidad para poner en valor la profesión, cuya práctica resulta aún insuficiente por la falta de formación de los profesionales en este campo, la limitada inversión de las compañías en investigación para su desarrollo, así como una carencia de tiempo por parte de los profesionales, centrados en tareas operativas (Castillo-Esparcia; Villabona-Beltrán, 2018).

\section{Profesionales y Academia: redefiniendo las competencias profesionales}

La gestión de la influencia resulta una de las especializaciones con mayor demanda en la actualidad, enmarcándose esta en las habilidades y competencias requeridas para los profesionales de la comunicación. Se trata, entre otras, de competencias vinculadas a la figura del Community Manager que apenas se recogen en los planes de estudios del área (Mañas-Viniegra; Jiménez-Gómez, 2019).

La brecha entre las demandas profesionales y la oferta formativa se enmarca en un contexto profesional definido por tres factores: la precariedad todavía es una constante (Mañas-Viniegra; Jiménez-Gómez, 2019), las fronteras entre disciplinas no siempre se plasman claramente entre los reclutadores (Álvarez-Flores; Núñez-Gómez; Olivares-Santamarina, 2018) y también la redefinición y actualización de roles no siempre es lo precisa que debiera (Silva-Robles, 2017) ya que con frecuencia las dinámicas de la realidad laboral avanzan varios pasos por delante de la conceptualización, como se aprecia en el auge de los chatbots (Serrano-Cobos, 2016).

Según el informe Global communications report (2017) sólo el 7\% de los trabajadores en agencia y el 12\% de los profesionales de departamentos de comunicación consideran que tendrán una función distinta o separada del departamento de marketing en los próximos años. Igualmente, el $87 \%$ de los profesionales considera que el término "relaciones públicas" no describirá su trabajo en los próximos cinco años. El mismo estudio, realizado en 2019, arroja que el 51\% de los profesionales percibe que el marketing y la comunicación estarán aún más integrados en los próximos cinco años. En el caso de las agencias, se identifica además la necesidad de crear departamentos especializados en I+D+i que engloben perfiles interdisciplinares (Carrillo-Durán, 2016).

El análisis de datos y la inclusión de la inteligencia artificial invita a la incorporación de profesionales de otros campos, que configuren equipos heterogéneos y contribuyan a integrar nuevos conocimientos, habilidades y competencias al área de la comunicación. Los estudios relacionados con el ámbito empresarial y la ingeniería computacional están ganando cada vez más protagonismo y marcan la diferencia en el desarrollo profesional. Igualmente, el reciclaje y la adaptación del talento en activo motivan el estudio continuo y el acercamiento a áreas distintas a las abordadas en la formación original.

La desinformación parece ser otra de las áreas que demandará una adaptación específica

La desinformación parece ser otra de las áreas que demandará una adaptación específica, aunque los profesionales no han evidenciado cambios significativos en sus rutinas de trabajo, ni han desarrollado, en la mayoría de los casos, pautas específicas para enfrentarse a ella (Rodríguez-Fernández, 2019).

Se añade, como fin último, generar interés en los nuevos profesionales para captar y retener el talento. Según el estudio 'La guerra por el talento', elaborado por la consultora de comunicación Llorente y Cuenca, el 38\% de los millennials confía en dejar su compañía en los próximos dos años y sólo el $40 \%$ se siente alineado con el propósito de su empresa. Igualmente, los estudiantes de titulaciones relacionadas manifiestan que los empleadores no tienen interés en ellos (Rodríguez-Fernández, 2018), punto de partida poco favorable para la futura contratación de estos egresados.

\section{Retos para la investigación en comunicación organizacional}

Pese a que en un entorno tan dinámico como el actual resulta complejo limitar los desafíos de la investigación de este ámbito, en esta sección se subrayan tres de los retos que pueden marcar los próximos años en relación a la comunicación organizacional: el diseño de un modelo longitudinal propio de investigación científica del ámbito profesional; la especialización funcional y competencial como complemento a la temática y la disciplinar; y un impulso a la investigación aplicada.

\section{1. Un modelo científico propio de aplicación al ámbito profesional}

En primer lugar, se hace necesario que la Academia desarrolle sus propios modelos de investigación introspectivos sobre el sector de la comunicación organizacional. Pese a que en los últimos años han emergido tímidamente algunos estudios específicos respecto a este ámbito desde la perspectiva científica, como el de Miquel-Segarra, López-Font y Gil-Soldevila (2018), se formula aquí la necesidad de plantear un diseño y un modelo sistemático que permita a la Universidad dotar a este campo de conocimiento de sus propios estudios. 
Una aproximación longitudinal que complemente las visiones transversales permitirá obtener una perspectiva panorámica que no solamente identifique tendencias actuales, sino que anticipe situaciones próximas, de modo que las aportaciones científicas puedan, siguiendo la filosofía que inspira el Plan Bolonia, continuar resultando útiles al ámbito profesional.
Se hace necesario que la Academia desarrolle sus propios modelos de investigación introspectivos sobre el sector de la comunicación organizacional

Evidentemente, todo ello no significa desdeñar las aportaciones que realizan en el ámbito nacional tanto asociaciones profesionales (Adecec, Dircom) como diversas consultoras (Evercom, Llorente y Cuenca, Torres y Carrera o Atrevia), que periódicamente presentan informes sobre el estado y tendencias del sector. Antes bien, sus propuestas constituyen un pilar de conocimiento imprescindible en la medida en que realizan una fotografía de proximidad.

Sin embargo, disponer de algún modelo de diseño independiente y con una metodología construida desde la exigencia científica serviría para consolidar las evoluciones que se registran en el ámbito profesional y, de este modo, contribuir desde las aulas a la preparación de los futuros empleados de la comunicación organizacional.

En ese sentido, propuestas como la de Túñez-López, Costa-Sánchez y Míguez-González (2018) constituyen un ejemplo de cómo, desde el rigor científico propio del ámbito académico, se puede realizar un diagnóstico a partir de la radiografía que arroja la situación profesional actual.

\subsection{De la especialización temática y disciplinar hacia la funcional}

Una aproximación global a la literatura científica de las últimas décadas permite comprobar que el interés de los investigadores ha seguido dos grandes líneas de trabajo: por una parte, la especialización temática en diferentes campos de actividad de los profesionales de la comunicación organizacional (política, financiera, económica, deportiva, médica...), incluso volviendo sus ojos a la propia representación pública de estos trabajadores (Sotelo-González; Cabezuelo Lorenzo, 2019).

Por la otra, bajo el paraguas de especialización disciplinar se recogen todas las investigaciones que se orientan a la definición de las diversas disciplinas o conceptos (o frentes, en palabras de Losada-Díaz, 2009) inherentes a la comunicación organizacional (comunicación interna, reputación, imagen, dirección de comunicación, relaciones con medios de comunicación, comunicación de crisis, responsabilidad social corporativa, branding, etc.).

De un modo paralelo y complementario a todo lo anterior, la revisión crítica de las competencias que exige el mercado profesional actual permitiría superar las limitaciones de los estudios que se adentran en un único campo de trabajo o en una sola disciplina. De hecho, la versatilidad técnica que en muchas ocasiones demandan los reclutadores son una prueba más de cómo este tipo de investigaciones podrían responder a esa necesidad al tiempo que se aporta un saber adicional al que emana de los estudios -en ocasiones solamente descriptivos- sobre el perfil académico o personal de los trabajadores de la comunicación corporativa.

La revisión crítica de las competencias que exige el mercado profesional actual permitiría superar las limitaciones de los estudios

\subsection{Un impulso a la investigación aplicada}

Sin renunciar a la incuestionable independencia de la investigación científica pura y a los imprescindibles que para el avance académico, profesional y social resultan los estudios que responden a los patrones de este modelo, se hace igualmente necesario reivindicar la investigación aplicada en el ámbito de la comunicación organizacional desde un triple punto de vista.

1) El auge de los estudios propios de las ciencias sociales que revelan las demandas de unos consumidores cuyo papel es cada vez más relevante en la conocida como generación 'Co', con quienes cabe esperar una actitud organizacional de simetría interactiva (Túñez-López; Costa-Sánchez; Míguez-González, 2019). Sus exigencias de comportamientos sostenibles y responsables acrecientan la necesidad de las entidades públicas y privadas de aprehender sus planteamientos a la hora de focalizar sus estrategias y tácticas de respuesta, como indica el previsible crecimiento de ámbitos como la responsabilidad social corporativa o el patrocinio y mecenazgo.

2) La riqueza de experiencias implementadas en la práctica profesional y diseminadas en los más variopintos campos de actividad también debe suponer un acicate al deseable auge de los estudios de caso que apliquen metodologías dispares y que vengan a enriquecer la investigación sobre comunicación organizacional. En ese sentido, el conjunto de estudios propios de este campo debe transitar con la misma facilidad por la vía inductiva y la deductiva, toda vez que el conocimiento teórico de este ámbito ha alcanzado un estado de madurez.

3) La investigación sobre comunicación organizacional puede abordar la encrucijada en la que se hallan organizaciones y públicos, que han sustituido (al menos desde el punto de vista teórico) su rol hegemónico tradicional de emisor y receptor por un sistema donde estos papeles se alternaban y, más recientemente, por el de cocreadores en un plano de aparente igualdad. 
En este punto existe una serie de preocupaciones compartidas (peligro de las fake news, dificultad de conocimiento real en entornos de infoxicación y de consumo multipantalla o las dinámicas de conexión en movilidad geolocalizada, entre otras) a cuyo desenlace la investigación científica también puede resultar útil.

\section{Conclusiones}

Reducir los retos profesionales y académicos en comunicación organizacional a las meras implicaciones tecnológicas supondría afrontar parcialmente una realidad volátil y en constante evolución. Por ello, cabe esperar que, más allá de coyunturas únicamente técnicas, la investigación focalice prioritariamente las consecuencias que se derivan de la adopción de estas innovaciones y que presentan un carácter más permanente.

En este sentido, la revisión y adaptación competencial de los profesionales del sector debe ir necesariamente acompañada de una conciencia de la centralidad del usuario, despojado de su rol como simple destinatario y que ahora adopta una relevancia a la altura de la que tiene la propia organización.

Academia y profesionales deben confluir a la hora de abordar críticamente las nuevas exigencias derivadas de un contexto donde la ética cobra todavía más protagonismo. Así, valores intangibles tradicionalmente cruciales como la confianza y la transparencia se reinterpretan ahora bajo la lupa de nuevos desafíos (posverdad, fake news, uso de datos personales, bots...) que, aun trascendiendo la comunicación organizacional, deben ser atendidos y entendidos por los estudiosos y los profesionales de esta disciplina ya que, inevitablemente, la están redefiniendo.

Finalmente, existen coordenadas como la hibridación a múltiples niveles (de equipos, de formatos, de géneros, de roles...), el engranaje de la investigación y la medición de resultados y la mercantilización de la información y la comunicación que pueden servir como claves para desentrañar los retos y desafíos que ha de afrontar la comunicación organizacional.

\section{Bibliografía}

Álvarez-Flores, Erika-Patricia; Núñez-Gómez, Patricia; Olivares-Santamarina, José P. (2018). “Perfiles profesionales y salidas laborales para graduados en Publicidad y Relaciones públicas: de la especialización a la hibridación”. El profesional de la información, v. 27, n. 1, pp. 136-147.

https://doi.org/10.3145/epi.2018.ene.13

Asociación de Directivos de Comunicación (2018). Anuario de la comunicación 2018. Madrid: Asociación de Directivos de Comunicación.

http://www.dircom.org/publicaciones/anuario/item/9153-anuario-de-la-comunicacion-2018

Asociación de Directivos de Comunicación (2018). El estado de la comunicación en España. Dircom.org. https://envios.dircom.org/docs/ECE-2018.pdf

Campos-Domínguez, Eva; García-Orosa, Berta (2018). “Comunicación algorítmica en los partidos políticos: automatización de producción y circulación de mensajes". El profesional de la información, v. 27, n. 4, pp. 769-777. https://doi.org/10.3145/epi.2018.jul.06

Carrillo-Durán, María-Victoria (2016). “Importancia del profesional de la información en la dirección de comunicación de las organizaciones". El profesional de la información, v. 25, n. 2, pp. 272-278.

https://doi.org/10.3145/epi.2016.mar.14

Castillo-Esparcia, Antonio; Villabona-Beltrán, Diego (2018). “Evaluación para demostrar la efectividad en comunicación y relaciones públicas. Una mirada a las prácticas profesionales desde los Communication Monitor". Communication papers, v. 7, n. 15, pp. 137-153.

http://ojs.udg.edu/index.php/CommunicationPapers/article/view/446/pdf

Cisco (2019). Cisco visual networking index: Forecast and trends, 2017-2022. White paper. https://www.cisco.com/c/en/us/solutions/collateral/service-provider/visual-networking-index-vni/white-paper-c11-741490. html

Gracia, Jordi (2017). "La posverdad no es mentir”. En: Ibáñez-Fanés, Jordi. La era de la posverdad. Barcelona: Criterios, p. 37. ISBN: 9788483594179

Han, Byung-Chul (2013). La sociedad de la transparencia. Barcelona: Herder. ISBN: 9788425432521

Hofeditz, Lennart; Ehnis, Christian; Bunker, Deborah; Brachten, Florian; Stieglitz, Stefan (2019). “Meaningful use of social bots? Possible applications in crisis communication during disassters". In: $27^{\text {th }}$ European conf on information systems (ECIS), Stockholm \& Uppsala.

https://www.researchgate.net/publication/332842989_MEANINGFUL_USE_OF_SOCIAL_BOTS_POSSIBLE_APPLICATIONS_ IN_CRISIS_COMMUNICATION_DURING_DISASTERS 
Llorente y Cuenca (2018). La guerra por el talento.

https://www.laguerraporeltalento.com

Losada-Díaz, José-Carlos (2009). "Los 10 frentes de la comunicación corporativa”. Doxa comunicación: revista interdisciplinar de estudios de comunicación y ciencias sociales, n. 9, pp. 145-164.

https://repositorioinstitucional.ceu.es/bitstream/10637/5896/1/n\%c2\%balX_pp145_164.pdf

Mañas-Viniegra, Luis; Jiménez-Gómez, Isidro (2019). “Evolución del perfil profesional del community manager durante la década 2009-2018". El profesional de la información, v. 28, n. 4.

https://doi.org/10.3145/epi.2019.jul.03

Miquel-Segarra, Susana; López-Font, Lorena; Gil-Soldevila, Samuel (2018). "Radiografía de las consultoras de comunicación en España: perfil profesional, estructura y actividad”. Revista latina de comunicación social, n. 73, pp. 478-503. https://doi.org/10.4185/RLCS-2018-1266

Redondo-Escudero, Myriam (2016). "Política automatizada: Bots, trols y propaganda digital encubierta en la comunicación internacional". ACOP Papers, n. 5, pp. 1-8.

https://compolitica.com/no5-myriam-redondopolitica-automatizada-bots-trols-y-propaganda-digital-encubierta-en-lacomunicacion-internacional

Rodríguez-Fernández, Leticia (2018). "Percepción de los estudiantes universitarios sobre el sector profesional de la comunicación corporativa”. Estudios sobre el mensaje periodístico, v. 24, n. 2, pp. 1677-1690.

https://doi.org/10.5209/ESMP.62240

Rodríguez-Fernández, Leticia (2019). “Desinformación: retos profesionales para el sector de la comunicación”. El profesional de la información, v. 28, n. 3.

https://doi.org/10.3145/epi.2019.may.06

Serrano-Cobos, Jorge (2016). "Tendencias tecnológicas en internet: hacia un cambio de paradigma". El profesional de la información, v. 25, n. 6, pp. 843-850.

https://doi.org/10.3145/epi.2016.nov.01

Silva-Robles, Carmen (2017). "The community manager: Responsibilities assigned by companies". En: Campos-Freire, Francisco; Rúas-Araujo, Xosé; Martínez-Fernández, Valentín-Alejandro; López-García, Xosé (2017). Media and metamedia management. Cham: Springer, pp. 271-277. ISBN: 9783319460680

https://doi.org/10.1007/978-3-319-46068-0_35

Sotelo-González, Joaquín; Cabezuelo-Lorenzo, Francisco (2019). “La representación del profesional de la comunicación política en el relato audiovisual contemporáneo”. En: Sierra-Sánchez, Javier; Lavín-de-la-Cavada, José-María. Redes sociales, tecnologías digitales y narrativas interactivas en la sociedad de la información. España: McGraw Hill, pp. 587-607. ISBN: 9788448618186

Túñez-López, José-Miguel; Costa-Sánchez, Carmen; Míguez-González, María-Isabel (2018). "Avances y retos de la gestión de la comunicación en el siglo XXI. Procesos, necesidades y carencias en el ámbito institucional". Estudios sobre el mensaje periodístico, v. 24, n. 1, pp. 921-940.

https://doi.org/10.5209/ESMP.59987

Túñez-López, José-Miguel; Toural-Bran, Carlos; Cacheiro-Requeijo, Santiago (2018). “Uso de bots y algoritmos para automatizar la redacción de noticias: percepción y actitudes de los periodistas en España". El profesional de la información, v. 27 , n. 4 , pp. $750-758$.

https://doi.org/10.3145/epi.2018.jul.04

USC Annenberg School for Communication and Journalism (2017). Global communications report 2017, annenberg.usc.edu. https://annenberg.usc.edu/sites/default/files/KOS_2017_GCP_April6.pdf

USC Annenberg School for Communication and Journalism (2019). Global communications report 2019, annenberg.usc.edu. http://assets.uscannenberg.org/docs/2019-global-communications-report.pdf

Wiencierz, Christian; Röttger, Ulrike (2019). "Big data in public relations: A conceptual framework". Public relations journal, v. 12, n. 3, pp. 1-15.

https://www.researchgate.net/publication/333220279_Big_Data_in_Public_Relations_A_Conceptual_Framework

Zerfass, Ansgar; Moreno, Ángeles; Tench, Ralph; Verčič, Dejan; Verhoeven, Piet (2017). European Communication Monitor 2017. How strategic communication deals with the challenges of visualisation, social bots and hypermodernity. Results of a survey in 50 Countries. Brussels: EACD/EUPRERA, Quadriga Media Berlin.

http://www.communicationmonitor.eu/2017/06/04/ecm-european-communication-monitor-2017-social-botsvisualisation-hypermodernity-benchmarking-strategic-communication 